Internist 2014 · 55:121-123

DOI 10.1007/s00108-013-3302-z

Online publiziert: 26. Januar 2014

๑) Springer-Verlag Berlin Heidelberg 2014

W.L. Gross ${ }^{1} \cdot$ S. Schreiber ${ }^{2}$

${ }^{1}$ Poliklinik für Rheumatologie, Universität zu Lübeck

${ }^{2}$ Klinik für Innere Medizin I, Universitätsklinikum Schleswig-Holstein, Kiel

\title{
Genetische Architektur chronisch-entzündlicher Systemerkrankungen
}

Nachdem kürzlich im Schwerpunkt „Systemische Inflammation " übergreifende pathogenetische Prinzipien und die Vielfalt inflammatorischer Reaktionsweisen im Fokus standen, soll in der vorliegenden Ausgabe deren genetischer Hintergrund beleuchtet werden: Können Risikoallele bei komplexen Erkrankungen zur Aufklärung von Pathomechanismen beitragen? Welchen Beitrag liefern Risikovarianten zur Präzisierung von „Diagnosen“ bei Systemerkrankungen? Wie ist der Einfluss exogener Risikofaktoren auf das Genom fassbar?

Die Nomenklatur von Systemerkrankungen ist uneinheitlich, die Diagnose basiert auf dem morphologischen Phänotyp und charakteristischen Labor, z. B. auf Autoantikörpern. Die Prognose ist oft unvorhersehbar und abhängig von krankheitspräzipitierenden bzw. reaktivierenden Umweltfaktoren. Immun- und Entzündungsreaktionen sind in Art und Umfang uneinheitlich. Die Genetik ist komplex, d. h meist nicht monogen-kausal, die Risikoprädiktion von Polymorphismen begrenzt. Einige Risikoallele sind mit Molekülen in Schaltstellen von Entzündung oder Stoffwechsel verknüpft und könnten damit zur tiefer greifenden Aufklärung pathogenetischer Abläufe beitragen.

\section{) Die Genetik von}

\section{Systemerkrankungen ist nur selten monogen-kausal}

Neue Sequenziertechniken führten zu modernen Genomstudien. Genomwei- te Assoziationsstudien (GWAS) haben Einzelnukleotidpolymorphismen ["single nucleotide polymorphisms" (SNP)] bei größeren Patientenkollektiven als Risikoallele erfasst. Deren Effektstärke ist im Einzelnen verhältnismäßig gering, aber in Risikofamilien durch die Risikoallelakkumulation erhöht. Mit dem „next generation sequencing" lassen sich auch kausale Varianten, eher seltene Mutationen, identifizieren.

Im Beitrag der Züricher Kollegen C. Ospelt u. S. Gay zur Bedeutung und den Mechanismen der Epigenetik lernen wir, wie der epigenetische Code durch verschiedene Umwelteinflüsse modifizierbar ist und wie diese Einfluss auf die Genexpression nehmen können. Mit den biochemisch darstellbaren Möglichkeiten der epigenetischen Modifikation, entweder direkt an der DNA oder an den Histonarmen, werden die Prinzipien der Anund Abschaltung von Genen vorgestellt. Mechanismen, die zur Chronifizierung von Entzündungen führen, werden dargelegt. Klinische Beispiele, u. a. die rheumatoide Arthritis, bei der die sehr frühe Behandlung eine Chronifizierung verhindern kann, werden vor dem epigenetischen Hintergrund dargestellt.

Komplexe Systemerkrankungen irritieren mit scheinbar gleichartigen Phänotypen, aber konträren Verlaufsformen, ähnlichen Immunphänomenen, aber unterschiedlichen pathogenetischen Prinzipien und sind somit diagnostisch und therapeutisch eine Herausforderung. Insofern sind die Einschlusskriterien für kontrollierte Studien (RCT) oft großzügig. Kön- 
nen moderne Genomanalysen Kranheitsentitäten zuverlässiger charakterisieren? Sind Risikoallele mit Kenngrößen der Immunpathogenese verknüpft und/oder sogar ätiologisch verantwortlich? Die Möglichkeiten werden exemplarisch von Lübecker u. Freiburger Kollegen für die Antineutrophile-zytoplasmatische-Antikörper(ANCA)-assoziierte Vaskulitis und die Sarkoidose vorgestellt.

Unter dem Terminus ANCA-assoziierte Vaskulitis werden klinisch und immunologisch definierte Kleingefäßvaskulitiden zusammengefasst und in kontrollierte Studien eingeschlossen. Die einzelnen „Varianten“, die Granulomatose mit Polyangiitis (GPA), die mikroskopische Polyangiitis (MPA) und die eosinophile Granulomatose mit Polyangiitis (EGPA), sind potenziell organ- bzw. lebensbedrohlich. Die pathognomonischen "pauci-immunen " Gefäßschäden, z. B. beim pulmorenalen Syndrom, sind wohl ANCA-induziert. Diese Autoantikörper sind gegen die Proteinase 3 (PR3-ANCA) oder die Myeloperoxidase (MPO-ANCA) gerichtet. Die MPA ist phänotypisch vaskulitisch geprägt, GPA und EGPA zeigen daneben auch granulomatöse Entzündungen, allerdings mit unterschiedlicher Histologie. Nach Clusteranalysen unterscheiden sich die "Varianten“ zudem im Verlauf.

In der GWAS zur ANCA-assoziierten Vaskulitis, in die GPA und MPA eingeschlossen wurden, konnten Risikoallele in Genbereichen für HLA-DPB1, PRTN3 (codiert PR3) und SERPINA1 [codiert $\alpha$ 1-Antitrypsin (a1AT), einen PR3-Inhibitor] identifiziert werden. Diese Risikogene waren allerdings nur mit der GPA assoziiert! GPA und MPA sind damit auch genetisch differenzierbare Entitäten! Die statistisch höhere Signifikanz der Risikogene mit dem ANCA-Status (PR3-ANCA), die stärker war als die für das klinische Syndrom (GPA), unterstreicht zusammen mit der Assoziation von „Autoimmunitätsallelen" von $H L A-D P B 1$ (GPA) und $H L A-D Q(\mathrm{MPA})$ die Autoimmunität. Interaktionstudien zu Produkten der Risikoallele, z. B. PRTN3 (codiert mit PR3 eine Serinproteinase) und SERPINA1 (codiert mit alAT den PR3-Inhibitor), werden mit Spannung erwartet.

Während der Verlauf der akuten Sarkoidose, des Löfgren-Syndroms, gutartig ist, ist die Prognose der chronischen Sarkoidose variabel. Die Bedeutung der Risikovarianten für den Verlauf und die bislang bekannten pathogenetischen Prinzipien werden vorgestellt. Dem mit dem Löfgren-Syndrom assoziierten $H L A$ DRB01*0301-Allel werden „nichtprotektive Allele" der chronischen Sarkoidose gegenübergestellt. Diese haben in Schweden Eingang in die Charakterisierung der Sarkoidose gefunden. Ferner werden Risikovarianten vorgestellt, die offenbar zu einer veränderten kostimulatorischen Signalgebung und überschießenden Immunreaktionen bzw. zu apoptotischen Abläufen führen oder in intrazelluläre Vorgänge des Molekülabbaus (Pathogene!) eingreifen.

\section{\) Die genetische Architektur der KHK umfasst mehr als 40 genetische Varianten}

Die Lübecker und Münchener Kollegen zeigen, dass die genetische Architektur der koronaren Herzerkrankung (KHK) mehr als 40 genetische Varianten umfasst. Dennoch hat sich die individuelle Risikoprädiktion durch die GWAS-Daten kaum verbessert. Die weitverbreiteten Polymorphismen sprechen für „eine tiefe Verwurzelung der Veranlagung für den Herzinfarkt in unserer Population" und können, akkumuliert in KHK-Familien, zu einer hohen Prävalenz von Infarkten führen. Kürzlich konnten die Autoren in einer Großfamilie mit der exomweiten Sequenzierung zwei extrem seltene infarktfördernde Mutationen identifizieren. Beide kausalen Gene führen zu einem Mangel an löslicher Guanylatcyclase und konsekutiv zu einer verminderten Aktivierung von cGMP, z. B. in Thrombozyten; tierexperimentell bewirken sie thrombotische Gefäßverschlüsse. Noch nicht betroffene Familienmitglieder können künftig gezielt mit Wirkstoffen zur Steigerung der Guanylatcyclase-Aktivität behandelt werden.

Alter und Geschlecht bleiben Hauptrisikoprädiktoren, traditionelle Risikofaktoren wie Hypertonie und Hypercholesterinämie erweitern sich durch Risikopatienten mit chronisch-entzündlichen Erkrankungen, z. B. mit Psoriasis oder rheumatoider Arthritis. Auf Verknüpfungsmöglichkeiten mit Schaltstellen des Stoffwechsels weist beispielsweise die Variante im SORT1-Genlokus hin. Sie induziert eine Veränderung der Bindungsstelle für den Transkriptionsfaktor C/EBP, reduziert den Sortilin-1-Spiegel und führt konsekutiv zu erhöhten LDL-Cholesterin-Werten. Dieser veränderte Signalweg im Fettstoffwechsel war zuvor nicht bekannt. Die Variante des ADAMTS7-Gens (codiert Metalloproteinasen) zeigt - nach Ausschaltung in Mäusen - einen KHKprotektiven Effekt. Andere SNP sind mit der Signaltransduktion von regulierenden Zytokinen [Interleukin(IL)-5, IL-6] assoziiert, die wahrscheinlich den entzündlichen Beitrag zur KHK dirigieren.

Aus dem Bereich der Dermatologie werden Genomstudien zu zwei Entzündungskrankheiten vorgestellt, dem atopischen Ekzem und der Psoriasis. Neben den Komorbiditäten sind in diesem $\mathrm{Zu}$ sammenhang die immunpathologisch geradezu gegenläufigen Immunreaktionen von Interesse. Das atopische Ekzem folgt einer Entzündung, die von Th2/Th22Zellen und den Zytokinen IL-4, IL-5 und IL-13 dominiert ist. Oft ist es mit Asthma bronchiale assoziiert. Die Psoriasis folgt einer Entzündung, die von Th1- und Th17-Zellen sowie von Interferon(IFN)- $\gamma$, IL-17, IL-21, IL-22 und IFN- $\alpha$ angetrieben wird. Vergesellschaftet ist die Psoriasis mit der Psoriasisarthritis und der KHK.

\section{I) Risikogene für entzündliche Dermatosen finden sich im epidermalen Differenzierungskomplex}

E. Rodríguez u. S. Weidinger aus Kiel fassen die in diversen Studien ermittelten erblichen Risikofaktoren zusammen und zeigen deren Assoziation mit den skizzierten T-Zell-Fehlsteuerungen, aber auch mit den Veränderungen in der epidermalen Funktion. Beim atopischen Ekzem stellt die Nullmutation des ProfillaggrinGens im epidermalen Differenzierungskomplex den stärksten bekannten Risikofaktor dar. Sie ist mit Verhornungsstörungen und Hauttrockenheit assoziiert. Die zur Krankheitsauslösung notwendigen ergänzenden Risikogene sind $\mathrm{z}$. T. ebenfalls 
im epidermalen Differenzierungskomplex oder auch in Zytokingenclustern (IL2/IL-21) lokalisiert. Das CARD14(NOD1)Allel weist zudem auf ein fehlgesteuertes NF- $\kappa B$ hin, einen Transkriptionsfaktor mit zentraler Funktion in der Inflammationsregulierung.

Bei der Psoriasis ist das HLA-Cw6-Allel der stärkste erbliche Prädispositionsfaktor. Risikogene finden sich ebenfalls im Bereich des epidermalen Differenzierungskomplexes, was auf die primäre Störung in der Hautbarriere hindeutet. Ergänzend ranken sich verschiedene Risikogene (UBLCP1/IL12B, EXOC2/IRF4) um die IL-23/Th17-Achse mit ihrer zentralen Rolle bei der kutanen Immunreaktion. Der therapeutisch verfügbare monoklonale Antikörper Ustekinumab ermöglicht durch die IL-12/23-Blockade eine sehr effektive Behandlung der Psoriasis und der Psoriasisarthritis.

Wie die Kollegen aus Kiel berichten, liegt die Zahl der Gene, die mit der Ätiologie chronisch-entzündlicher Darmerkrankungen - Colitis ulcerosa und M. Crohn - assoziiert sind, mittlerweile bei über 190. Für viele der identifizierten Krankheitsgene und krankheitsassoziierten Regionen bestehen multiple Verbindungen zu anderen entzündlichen und auch nichtentzündlichen Erkrankungen, bei denen die gestörte Interaktion zwischen Darmbarriere und mikrobieller Umwelt eine grundlegende Bedeutung hat.

Der enorme Fortschritt auf diesem Wissensgebiet wird auch zu neuen Herausforderungen an die Medizin führen.
Die Promiskuität der Krankheitsgene über die Indikationsgrenzen hinweg weist darauf hin, dass die Systemmedizin von morgen neuen Einteilungen folgen muss, um die sich aus den genetischen und genomischen Erkenntnissen entwickelnden Prinzipien in Diagnostik und Therapie umsetzen zu können.

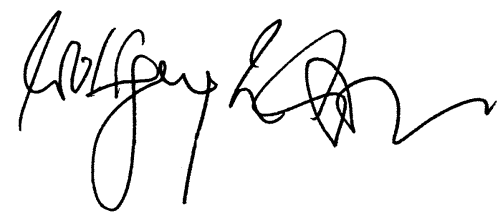

W. Gross
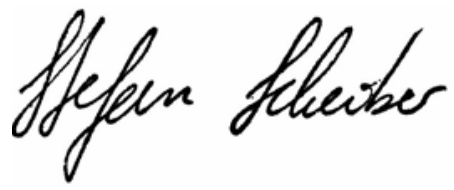

S. Schreiber

\section{Korrespondenzadresse}

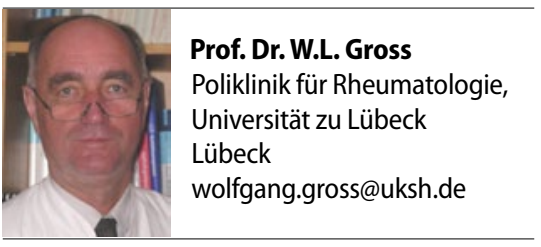

\section{Einhaltung ethischer Richtlinien}

Interessenkonflikt. W.L. Gross hat von Roche finanzielle Zuwendungen (Referententätigkeit, Reisekostenübernahme) erhalten und von GSK ebenso Zuwendungen für Beratungstätigkeiten bekommen. S. Schreiber gibt an, dass kein Interessenkonflikt besteht.

\section{Immundefektsyndrome}

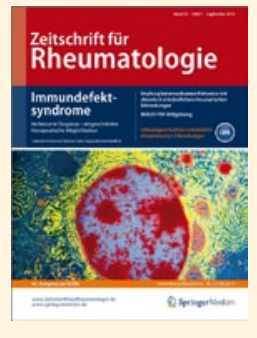

Die gerade auch in Deutschland sehr aktive Immundefektforschung hat in den vergangenen Jahren zahlreiche neue Gendefekte als Ursache primärer Immund-

efizienzen charakterisiert. Den wesentlich verbesserten diagnostischen Möglichkeiten stehen derzeit jedoch leider noch immer nur begrenzte therapeutische Möglichkeiten gegenüber. Die Beiträge der Ausgabe 7/2013 der Zeitschrift für Rheumatologie liefern einen breiten, aktuellen Überblick über Ätiologie, Pathophysiologie, Diagnostik und Therapie von primären und sekundären Immundefektsyndromen. Lesen Sie über folgende Themen ausführlicher:

- Sekundäre Immundefizienz bei rheumatologischen Erkrankungen

- Angeborene Immundefekte

- Variabler Immundefekt

- Primäre und sekundäre Neutropenie

Bestellen Sie diese Ausgabe zum Preis von 36,- Euro zzgl. Versandkosten bei

Springer Customer Service Center Kundenservice Zeitschriften Haberstrasse 7

69126 Heidelberg

Tel.: +49 6221-345-4303

Fax: +49 6221-345-4229

E-Mail: leserservice@springer.com

Suchen Sie noch mehr zum Thema? Mit e.Med, dem Online-Paket von Springer Medizin, können Sie schnell und komfortabel in über 500 medizinischen Fachzeitschriften recherchieren.

Weitere Infos unter springermedizin.de/eMed. 